\title{
Short-term Fluctuations Of The Mesospheric Na Layer Observed By High-time-resolution Lidar
}

\author{
Xiaodong Wang ${ }^{1, *}$, Fan $\mathrm{Yi}^{2}$ \\ ${ }^{1}$ The 38th Research Institution of China Electronics Technology Group \\ ${ }^{2}$ School of Electronic Information, Wuhan University, Wuhan, China.
}

\begin{abstract}
Based on the 6-s resolution Na lidar measurements during 2395 hours on 47 different nights from May to November 2011 in Beijing $\left(40.2^{\circ} \mathrm{N}, 116^{\circ} \mathrm{E}\right)$, China, it was found that the $\mathrm{Na}$ density at altitude 83-98 km always exhibited strong short-term fluctuations. The magnitude of the mean absolute increasing and decreasing rates for these short-term fluctuations ranged from $\sim 8$ to $\sim 16 \mathrm{~cm}^{-3} \mathrm{~s}^{-1}$. Their profiles were close to each other with the increasing rate being slightly larger than that of decreasing rate at most altitudes. This difference coincided with the earlier observations that the Na layer column abundance mostly tends to have a slow net increase during night [2]. The characteristic time for the short-term $\mathrm{Na}$ density fluctuations had a magnitude ranging from 46 to $118 \mathrm{~s}$ at altitudes between 83 and $98 \mathrm{~km}$, which was apparently shorter than the Brunt-Väisälä period ( $\sim 5 \mathrm{~min})$. The gravity wave seems difficultly to induce observed $\mathrm{Na}$ fluctuations.
\end{abstract}

\section{Introduction}

Free metal atom layers exist in the mesosphere and lower thermosphere (MLT) from 80 to $110 \mathrm{~km}$. They are widely believed to be originated from meteor ablation $[2$, 4, 23]. Extraterrestrial micrometeoroids enter the Earth atmosphere with speed between 11 and $72 \mathrm{kms}^{-1}$, and ablate in the mesosphere and lower thermosphere [12, 24]. According to the modeling by Plane [23], the slow meteors $\left(\sim 18 \mathrm{kms}^{-1}\right)$ have their maximum ablation efficiency around $90 \mathrm{~km}$, fast ones $\left(>\sim 40 \mathrm{kms}^{-1}\right)$ are shifted towards $100 \mathrm{~km}$ or even higher into the ionosphere given that the incoming micrometeoroids have a median mass of $10 \mu \mathrm{g}$ and median radius of 100 $\mu \mathrm{m}$. The input flux of micrometeoroids into the atmosphere is rather uncertain, because no single technique can observe particles over the mass range from about $10-12$ to $1 \mathrm{~g}$, which makes up the bulk of the incoming material [1]. Zodiacal cloud observations [22] and measurements made with a spaceborne dust detector [19] indicate a daily mass input of interplanetary dust particles ranging from 100 to 300 tonnes, which is in agreement with the accumulation rates of cosmicenriched elements ( $\mathrm{Ir}, \mathrm{Pt}$, Os and super-paramagnetic $\mathrm{Fe}$ ) in polar ice cores and deep-sea sedimentations $[8,14,15]$. In contrast, measurements in the middle altitude atmosphere - by radar [20], lidar [11, 25], high-flying aircraft [6] and satellite remote sensing [13] indicate that the input is between 5 and 50 tonnes per day.

Although metal atomic layer has been studied for about forty years, there are still some core problems unanswered in understanding the entire chain of processes and reactions about its origin of the normal metal layers, in particular the magnitude of the gasphased metal injection rate [26]. Firstly, there is still no direct measurement of the total injection flux by meteoric ablation. Secondly, temperature at the edge of meteor trail was $20-50 \mathrm{~K}$ warmer than that at the trail tube center and surrounding atmosphere around 3 minutes after ablation [3], which could not get satisfactory explanation in both chemical and frictional heating $[21,27]$. Thirdly, mechanisms of the sporadic layers reported in literature and their relation with permanent main layer is still mysterious [28]. Furthermore, a vital and unresolved question is whether metal atoms could be treated as stable tracers to study the atmospheric waves in the mesosphere and lower thermosphere [29]. It depends on the lifetime of individual metal atoms.

The characteristic time (time constant) for the density variation of lidar-observed metal atoms is important for accurately understanding their fundamental processes (e.g., gas-phase metal injection from meteoric ablation, gas-phase chemistry, condensation and coagulation, dynamic transport and sedimentation). Its magnitude can help researchers to identify what processes are taking place. In terms of lidar-measured $\mathrm{Na}$ number density $\rho_{N a}(r, t)$, the transient characteristic time is defined as Chen and $\mathrm{Yi}[2]$

$$
\tau=\left[\left(\frac{\partial \rho_{N a}(r, t)}{\partial t}\right) / \overline{\rho_{N a}(r, t)}\right]^{-1}=\left\{\frac{\rho_{N a}(r, t+\Delta t)-\rho_{N a}(r, t)}{0.5\left[\rho_{N a}(r, t+\Delta t)+\rho_{N a}(r, t)\right] \Delta t}\right\}^{-1}
$$

Where, $\Delta \mathrm{t}$ is time resolution for lidar measurement, the average $\mathrm{Na}$ density in the middle equation is the mean value between two successive measurements. The calculated characteristic time (time constant) from the 5min-resolution lidar measurements ranges from $\sim 0.07$ to

\footnotetext{
* Corresponding author: wxd@whu.edu.cn
} 
$\sim 2.0 \mathrm{~h}$ for Na main layer and from $\sim 0.02$ to $\sim 1.7 \mathrm{~h}$ for $\mathrm{Fe}$ main layer [2]. The observational analysis on sporadic $\mathrm{Na}$ layer showed that larger $\mathrm{Na}$ peak densities were obtained with a lidar with shorter time resolution $(8 \mathrm{~s})$ [16]. It was found that each of the observed sporadic $\mathrm{Na}$ layers consisted of a sequence of small-timescale (16112 s) density enhancement bursts [16]. In addition, the peak height of a sporadic Na layer was observed to be slightly different for different 1.5 -s lidar profiles within a time period of $5 \mathrm{~min}$ [17]. A long integration period would not only underestimate the apparent change rates of $\mathrm{Na}$ atomic density, but also smooth out the fine structures of Na layer, thus impeding our understanding of the actual Na behavior on small time scale [17].

Based on the $6 \mathrm{~s}$ time-resolution Na lidar data (47 nights from May to November 2011) observed at Yanqing, $\left(115.97^{\circ} \mathrm{E}, 40.47^{\circ} \mathrm{N}\right)$ Beijing, China, we present in this paper the rate and characteristic time for the $\mathrm{Na}$ density variation for the $\mathrm{Na}$ main layer at different altitudes. The primary terms in the $\mathrm{Na}$ continuity equation are evaluated respectively under normal MLT conditions and compared with the lidarmeasured change rates. Based on a scale analysis of the $\mathrm{Na}$ continuity equation, the large $\mathrm{Na}$ transient change (increasing and decreasing) rate observed by lidar (rapid $\mathrm{Na}$ fluctuation) might be attributed to direct meteoric $\mathrm{Na}$ input and some removal processes (e.g., recondensation, coagulation and sedimentation).

\section{Instrument and data description}

The data utilized in this paper come from the $\mathrm{Na}$ resonance fluorescence lidar measurement at Yanqing, $(115.97 \mathrm{oE}, 40.47 \mathrm{oN})$ Beijing, China. The lidar was set up in November 2009 under the support of the Chinese Meridional Project [17, 28]. Its transmitter is a tunable dye layer pumped by a frequency-doubled Nd: YAG laser with a pulse repetition rate of $30 \mathrm{~Hz}$. The dye laser allows a rapid and convenient selection of transmitted wavelength at the beginning of each lidar observation night. With a help of a $\mathrm{Na}$ vapor cell, the pulsed dye laser is finely tuned and stabilized at the D2 resonant absorption line of $\mathrm{Na}$. The laser emits an output energy of $60 \mathrm{~mJ}$ at each 8 -ns pulse. The laser beam has a linewidth of $1.8 \pm 0.3 \mathrm{GHz}$ and is expanded with a 5 beam expander. The resulting beam is of a divergence of

$0.5 \mathrm{mrad}$. The expanded laser beam is then transmitted vertically into the atmosphere by a computer-controlled gimbal-mounted mirror. The backscattered photons are collected by a receiving telescope with a $1-\mathrm{m}$ diameter primary mirror. A fan-cooled photomultiplier tube is used for the photon detection. The output pulses from the PMT are fed into a multi-channel scaler (MCS) controlled by a PC. In order to study the small-scale change of $\mathrm{Na}$, the temporal resolution is set to $1.5 \mathrm{~s}$ with

$0.83 \mathrm{~s}$ for laser shot accumulation (25 shots) and

$0.67 \mathrm{~s}$ for data transfer. The time bin length taken here is 640 nanoseconds, corresponding to a vertical resolution of $96 \mathrm{~m}$. Sporadic Na layers [4-5] were observed at the Beijing lidar site near 40o N [17; 28]. Since the present study is concerned with only the small- scale variations of the main $\mathrm{Na}$ layer, the profiles containing such sporadic layers have been excluded from $\mathrm{Na}$ data (in terms of the criteria of sporadic Na layer) [45]. The remaining data represent $\sim 396 \mathrm{~h} \quad \mathrm{Na}$ measurements on 47 different nights from May to November in 2011. The data duration per night is larger than $5 \mathrm{~h}$. In order to reduce the measurement error for $\mathrm{Na}$ density, the raw $1.5 \mathrm{~s}$ data (25 shots) are integrated to yield 6-s photon count profiles. The Na density profiles are derived by using a standard method with reference altitude $30 \mathrm{~km} \mathrm{[10].} \mathrm{For} \mathrm{a} \mathrm{shorter} \mathrm{temporal} \mathrm{resolution}$ (integration period), the random measurement error for $\mathrm{Na}$ density increases. Thus an error estimate is needed to assess the reliability of the $\mathrm{Na}$ density fluctuations. The random error for the lidar-measured $\mathrm{Na}$ density results primarily from fluctuations in the signal levels. A general expression for the random error was firstly introduced by Gardner et al., 1986 [9] and then simplified by Gardner, 1989[10]

$$
\operatorname{Std}\left[n_{s}(z)\right]=\left[C_{s} n_{s}(z) / N_{s} \Delta z\right]^{1 / 2}
$$

Where $C_{\mathrm{s}}$ is Na column abundance, $N_{\mathrm{s}}(\mathrm{z})$ is $\mathrm{Na}$ density at altitude $\mathrm{z}, N_{\mathrm{s}}$ is the total photon count comprising the Na profile and $\Delta z$ is the range bin length. This expression is used to estimate the Na measurement error in this work.

\section{Observational results}

As an example, Figures $1 \mathrm{a}$ and $1 \mathrm{~b}$ present the contour plots of $\mathrm{Na}$ density measured on the night of 2 October 2011 with time resolutions of $6 \mathrm{~s}$ and 5 min respectively. The 6-s Na density contour on this night showed significant small-scale fluctuations with nearly vertical plumed structure visible on the top and bottom of the $\mathrm{Na}$ layer (as shown in Figure 1a). The Na variation shown in the 5-min density contour (Figure $1 \mathrm{~b}$ ) was apparently more moderate and smooth compared to that in the 6-s density contour. The peak density had a maximum of $4321 \pm 160 \mathrm{~cm}-3$ at $89.0 \mathrm{~km}$ and 20:25:57 LT for the 6-s $\mathrm{Na}$ data, while the corresponding maximum was $4010 \pm 25 \mathrm{~cm}-3$ at $90.5 \mathrm{~km}$ for 5 -min data. The coarse time resolution adopted $(5 \mathrm{~min})$ gave rise to an underestimate of the maximum $\mathrm{Na}$ peak density as mentioned by Liu and Yi [16] and Liu et al. [17]. Figure 2 gives an example of the temporal variations of the calculated absolute and relative Na variation rates at $92-$ $\mathrm{km}$ altitude over 10 minutes (from 1853 to $1903 \mathrm{LT}$ ) on the night of 2 October 2011 at Beijing, China. The absolute transient increasing and decreasing rates had the average values of $18.2 / 15.3 \mathrm{~cm}^{-3} \mathrm{~s}^{-1}$ over the 10 minutes, while the corresponding mean values for the relative rates were $0.0054 / 0.0045 \mathrm{~s}^{-1}$.

The estimated characteristic time (time constant) for the $\mathrm{Na}$ transient variation is $\sim 202$ s. Apparently, the increasing and decreasing rates represented the magnitude of local $\mathrm{Na}$ density fluctuations which reflected local $\mathrm{Na}$ atom production/removal or advection of small-scale Na horizontal structures by large-scale wind or turbulence. Since the value of the characteristic 
time was less than the buoyancy period $(5 \mathrm{~min})$ in the mesopause region [7], these small-scale Na fluctuations were not due to atmospheric gravity waves.
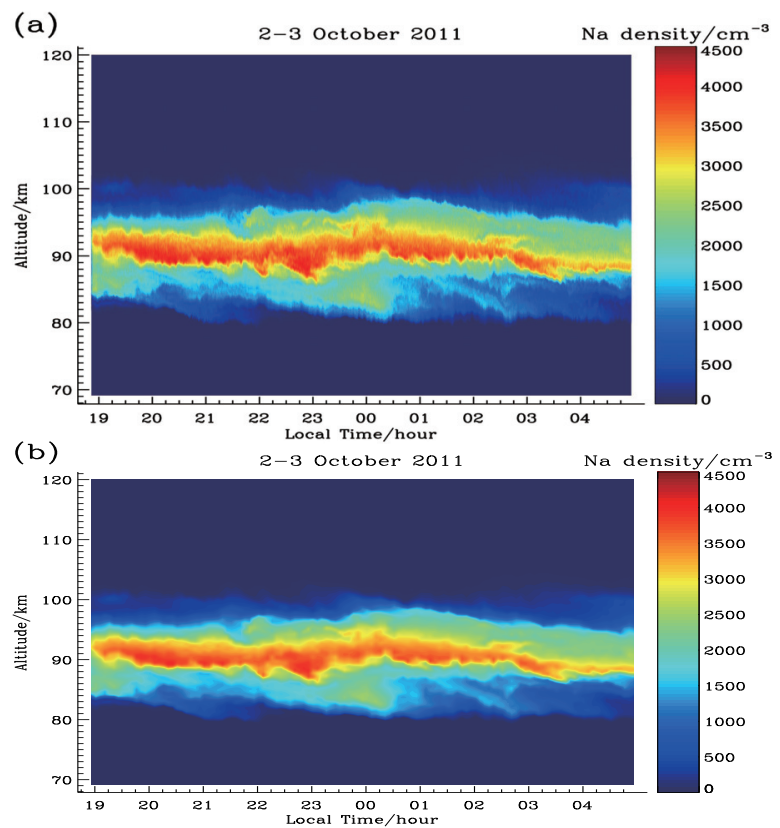

Fig. 1. Contour plots of $\mathrm{Na}$ density measured by a $\mathrm{Na}$ lidar at Yanqing, Beijing, China on the night of 2 October 2011 with different time resolutions: (a) $6 \mathrm{~s}$ and (b) $5 \mathrm{~min}$. The height resolution is $96 \mathrm{~m}$ for both plots.

(a)

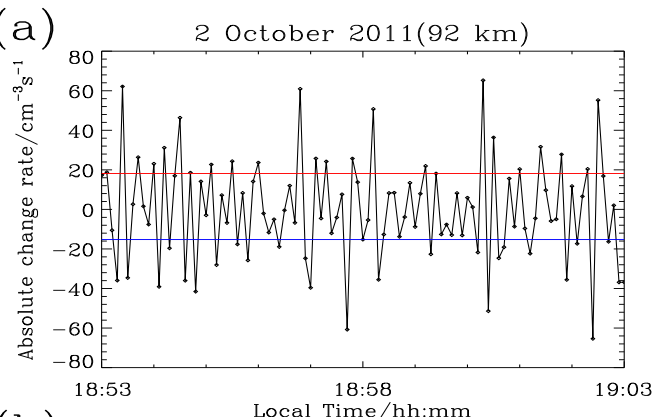

(b)

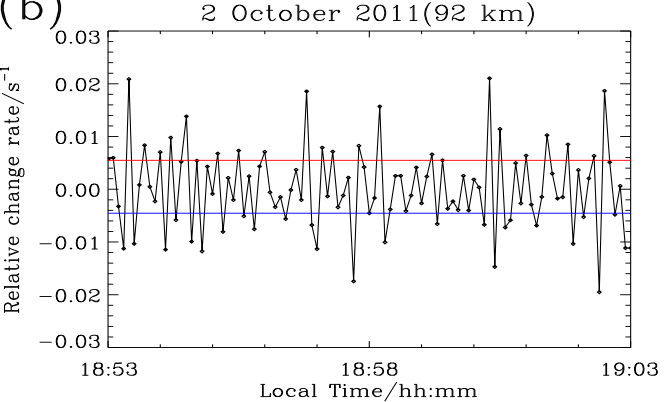

Fig. 2. Temporal variations of the absolute (a) and relative (b) $\mathrm{Na}$ variation rates measured at $92 \mathrm{~km}$ from 18:53 to 19:03 LT on the night of 2 October 2011 with a time resolution of $6 \mathrm{~s}$.

Then all the lidar data from 1 May to 27 November 2011 totally 47 days with $6 \mathrm{~s}$ time resolution were investigated. The mean profiles of change rate were calculated and shown in Figure 3. The profiles were derived with the same method proposed by Chen and Yi [2]: (1) Absolute change rate profiles for a given night are obtained via a difference calculation with two adjacent 6-s density profiles. At a given altitude, the positive and negative rate values were respectively gathered together to form increasing and decreasing rates data subsets. The mean increasing and decreasing rates were attained by respectively taking the medians of the two rate subsets. The calculated results at all altitudes on this night were put together to produce the nightly mean profiles of the increasing and decreasing rates. (2) The overall mean profiles of the increasing and decreasing rates were acquired by taking the medians of the 47 nightly mean profiles for the increasing and decreasing rates respectively. Note that a median value was employed instead of an arithmetic mean in all average calculations because it less affected by possible extreme rate values. As seen in Figure 3a, the mean absolute $\mathrm{Na}$ increasing and decreasing rates on the $\mathrm{Na}$ main layer were altitude dependent and had a single peak structure. Their values were larger than $10 \mathrm{~cm}^{-3} \mathrm{~s}^{-1}$ at altitudes between 84 and $97 \mathrm{~km}$ with a maximum of $16 \mathrm{~cm}^{-3} \mathrm{~s}^{-1}$ near $90 \mathrm{~km}$ altitude. The difference between absolute increasing and decreasing rates ranged from 0.01 to 0.17 $\mathrm{cm}^{-3} \mathrm{~s}^{-1}$ (83 to $98 \mathrm{~km}$ ). In terms of the mean relative $\mathrm{Na}$ increasing and decreasing rates shown in Figure $3 \mathrm{~b}$, the characteristic time or time constant (reciprocal of relative change rate) had a maximum of $\sim 120 \mathrm{~s}$ at 90.5 $\mathrm{km}$, which was much smaller than that value of $\sim 2.0 \mathrm{~h}$ obtained from the 5 minute $\mathrm{Na}$ profiles by Chen and $\mathrm{Yi}$ [2]. We note that mean change rates (both absolute and relative) over the 47 nights were in magnitude and altitude dependence very similar to that for the individual night. This implies that the small-scale $\mathrm{Na}$ density fluctuations are steady from day to day. Interestingly, the peak altitudes for the $\mathrm{Na}$ density increasing and decreasing rates were close to that of the background $\mathrm{Na}$ layer. The magnitudes of the observed change rates and characteristic time for $\mathrm{Na}$ atoms were significant for evaluating the roles of various factors or mechanisms (e.g., metal deposition from meteor ablation, chemical reactions, dynamical processes and so on) in the observed small-scale $\mathrm{Na}$ fluctuations.

\section{Summary and conclusions}

Rapid $\mathrm{Na}$ density fluctuations in the mesospheric $\mathrm{Na}$ layer had been investigated based on the 6-s resolution $\mathrm{Na}$ lidar measurements during $\sim 395$ hours on 47 different nights from May and November 2011 in Beijing $\left(40.2^{\circ} \mathrm{N}, 116^{\circ} \mathrm{E}\right)$, China. It was found that the $\mathrm{Na}$ density at any altitude of the $\mathrm{Na}$ main layer always exhibited significant short-term fluctuations. The mean absolute increasing and decreasing rate profiles for the short-term $\mathrm{Na}$ density fluctuations had a single-peak structure with values ranging from 8 to $16 \mathrm{~cm}^{-3} \mathrm{~s}^{-1}$ on the $\mathrm{Na}$ main layer (peak value of 16.2 atoms $\mathrm{cm}^{-3} \mathrm{~s}^{-1}$ at $\sim 92$ $\mathrm{km})$. This structure was rather similar to the background $\mathrm{Na}$ density profile. The mean profiles of the local transient $\mathrm{Na}$ increasing and decreasing rates were close to each other, but the values of the increasing rate were slightly larger than that of the decreasing rate at most altitudes. The positive small difference $\left(0.01\right.$ to $0.17 \mathrm{~cm}^{-}$ ${ }^{3} \mathrm{~s}^{-1}$ ) at most 

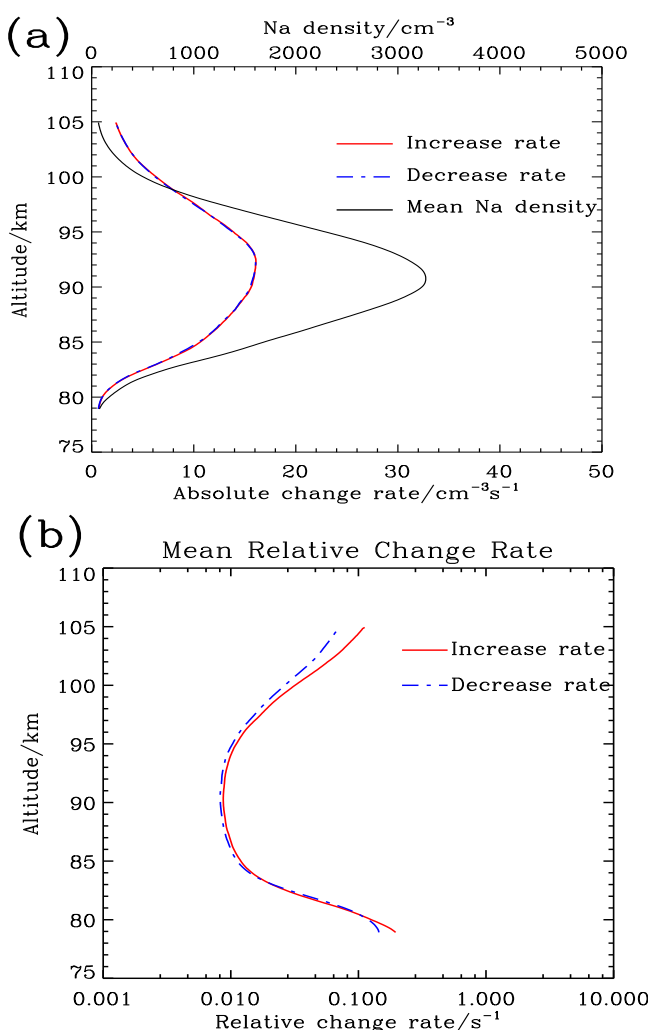

Fig. 3. Profiles of the mean absolute (a) and relative (b) $\mathrm{Na}$ increasing and decreasing rates from the 47 nights of Na lidar measurements (from May to November 2011) at Yanqing.

altitudes coincided with previous observations that the Na layer column abundance mostly tends to have a slow net increase during night (because the $\mathrm{Na}$ lidar data were collected only during night) [2]. The characteristic time (time constant) for the short-term $\mathrm{Na}$ density fluctuations was calculated in terms of a conventional method. Its magnitude ranged from 46 to $118 \mathrm{~s}$ at altitudes between 83 and $98 \mathrm{~km}$, which was apparently shorter than the Brunt-Väisälä period (5 min). This result eliminated a possibility that gravity waves were responsible for the short-term Na density fluctuations.

\section{Acknowledgement}

The Yanqing Na lidar in Beijing, China was supported by the Meridian Space Weather Monitoring Project. This research is supported jointly by the National Natural Science Foundation of China through grants 41404120 and 41327801 and Ocean Public Welfare Scientific Research Project from State Oceanic Administration, People's Republic of China (No. 201205036).

\section{References}

1. Ceplecha, Z., Borovička, J., Elford, W. G., et. al., Space Sci. Rev. 84(3-4), 327-471, (1998)

2. Chen, L., and Yi, F., Ann. Geophys. 29, 1037-1048, (2011)

3. Chu, X., Liu, A. Z., Papen, G., et. al., Geophys. Res. Lett. 27(13), 1815-1818, (2000)
4. Clemesha, B. R., V. Kirchhoff, D. M. Simonich, et al., Geophys. Res. Lett. 5, 873-876, (1978)

5. Clemesha, B. R., J. Atmos. Sol-Teer. Phy. 57(7), 725-736, (1995)

6. Cziczo, D. J., Thomson, D. S., and Murphy, D. M., Science, 291(5509), 1772-1775, (2001)

7. Fritts, D. C., Alexander, M. J., Rev. of Geophys. J41(1), ( 2003)

8. Gabrielli, P., C. Barbante, J. M. C. Plane et al., Nature 432, (2004)

9. Gardner, C. S., D. G. Voelz, et. al., Journal of Geophysical Research 91(A12), 13659-13673, (1986)

10. Gardner, C. S., Proceeding of the IEEE 77(3), 408418, (1989)

11. Gardner, C. S., X. Z. Chu, et. al., J. Geophys. Res. 116, D02304

12. Gardner, C. S., A. Z. Liu, D. R. Marsh, W. Feng, and J. M. C. Plane, J. Geophys. Res. 119, 78707879

13. Hervig, M. E., L. L. Gordley et al., Geophys. Res. Lett. 36, L18805, (2009)

14. Lanci, L. and D. V. Kent, Geophys. Res. Lett. 33, L13308, (2006)

15. Lanci, L., Kent D. V., and Biscaye P. E., Geophys. Res. Lett. 34, L10803, (2007)

16. Liu, Y. J., F. Yi, J. Atmos. Sol-Teer. Phy., 71(12), 1374-1382, (2009)

17. Liu, Y. J., Clemesha, B. R., Wang, J. H., and Cheng, X. W, Ann. Geophys. 31, 1899-1912, (2013)

18. Liu, Y. J., Plane, J. M. C., Clemesha, B. R., et al., Ann. Geophys. 32(10), 1321-1332, (2014)

19. Love S. G, Brownlee D. E., Science 262(5133): 550-553, (1993)

20. Matthews, J. D., Janches, D. et. al., Geophys. Res. Lett. 28(10), 1929-1932, (2001)

21. Nesse, H., D. Heinrich et. al., Ann. Geophys. 26(5), 1071-1081, (2008)

22. Nesvorny D., Jenniskens P. et. al., Astrophys. J. 713, 816-836, (2010)

23. Plane, J. M. C., R. M. Cox, and R. J. Rollason, Adv. Space Rev. 24(11), 1559-1570, (1999)

24. Plane J. M. C., Che. Rev. 103(12), 4963-4984, (2003)

25. Plane, J. M. C., Atmos. Che. Phy. 4, 627-638, (2004)

26. Plane, J. M. C., Wuhu Feng, and Erin C. M. Dawkins, Che. Rev. 20, 0009-2665, (2015)

27. Qian, J. and C. S. Gardner, Journal of Geophysical Research 100(D4), 7453-7461, (1995)

28. Wang, J., Y. Yang, X. Cheng, G. Yang, S. Song, and S. Gong, Geophys. Res. Lett. 39, L15801, (2012)

29. Xu, J., and A. K. Smith, Geophys. Res. Lett. 30, 2056, (2003) 